\title{
TMSCl mediated highly efficient one-pot synthesis of octahydroquinazolinone and 1,8-dioxo-octahydroxanthene derivatives
}

\author{
Srinivas Kantevari,* Rajashaker Bantu, and Lingaiah Nagarapu \\ Organic Chemistry Division-II, Indian Institute of Chemical Technology, Uppal Road, \\ Hyderabad-500007, India \\ E-mail: Kantevari@yahoo.com
}

\begin{abstract}
A simple, efficient and cost-effective method for the synthesis of octahydroquinazolinone and 1,8-dioxo-octahydroxanthene derivatives by a one-pot cyclocondensation of dimedone and aldehydes, with and without urea or thiourea respectively in the presence of Trimethylsilyl chloride (TMSCl) in MeCN/DMF has been described.
\end{abstract}

Keywords: Octahydroquinazolinone, trimethylsilyl chloride, 1,8-dioxo-octahydro xanthene, cyclocondensation, one pot multi-component synthesis

\section{Introduction}

Octahydroquinazolinone derivatives have attracted considerable attention in recent years owing to their potential antibacterial activity against Staphylococcus aureus, Escherichiacoli, pseudomonas aeruginosa ${ }^{1}$, and also as a calcium antagonist ${ }^{2}$. Methods employed for their synthesis are the variant classical one pot multi-component Biginelli reaction involving dimedone, aromatic aldehydes and urea $^{3}$. Although various Lewis acid catalysts are employed $^{1,3,4 \mathrm{a}-\mathrm{c}}$ in the extension of the Biginelli reaction, they are expensive, harmful and are difficult to handle especially on large scale. Most of these procedures are sluggish, require longer reaction times, use strongly acidic conditions, give unsatisfactory yields and also suffer from the formation of many side products $4 \mathrm{a}$.

In continuation of our work on the use of silica-supported reagents ${ }^{5}$, TMSCl has attracted our interest. Among the various silicon-based acidic reagents, TMSCl has received considerable attention as an inexpensive and readily available reagent for various organic transformations ${ }^{6}$. Advantages such as its compatibility with many synthetically valuable nucleophiles (e.g., organometalic reagents, and cuprates) and its non-aggregation nature substantially simplify the analysis of the reaction mechanisms. Because of this, it has been extensively used as a protecting 
group for various functional groups such as hydroxy and amino group ${ }^{6 a, 7}$ and as a promoter for cycloadddition and conjugate addition reactions ${ }^{6 a}$ under mild and convenient conditions to offer the products in excellent yield and high selectivity. However, to the best of our knowledge, there is no report on the synthesis of octahydroquinazolinone using TMSCl as a reagent. In this communication, we report a TMSCl mediated simple, efficient and environmentally benign synthesis of octahydroquinazolinone (Schemes $1 \& 2$ ). During our study we also observed the formation of 1,8-dioxo-octahydroxanthene in excellent yields by one-pot Knoevenagel condensation, Michael addition and cyclodehydration of dimedone with various aldehydes in the presence of TMSCl (Scheme 3).

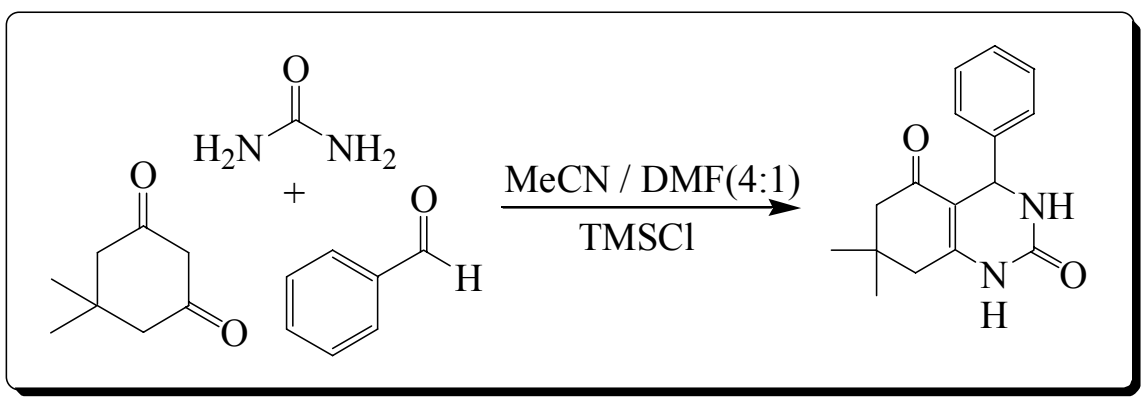

\section{Scheme 1}

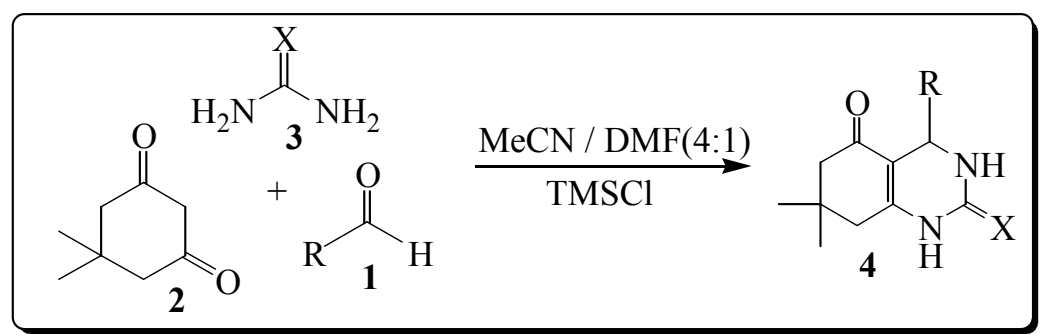

\section{Scheme 2}

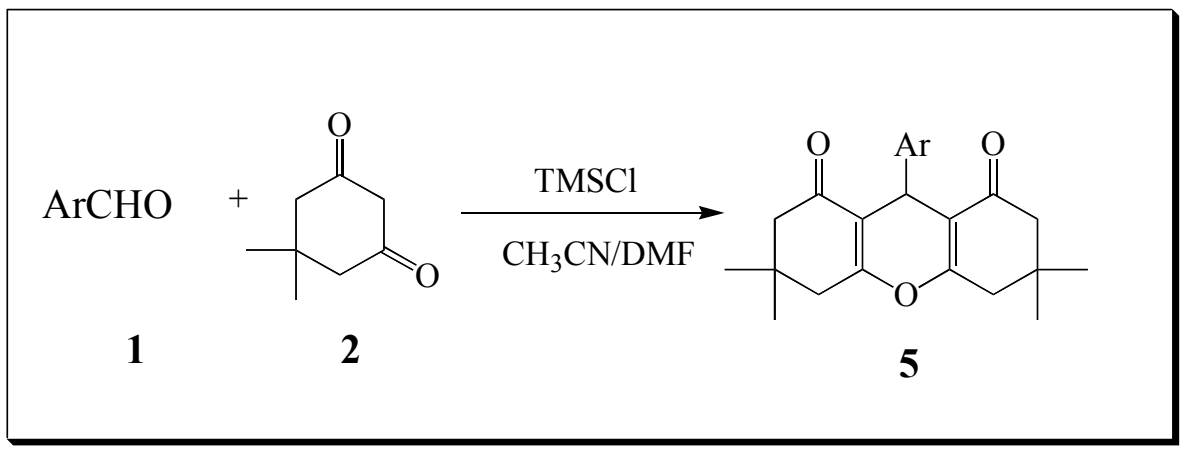

Scheme 3 
Initially, a pilot reaction was attempted using benzaldehyde (1), dimedone (2) and urea (3) in the presence of TMSCl ( 0.5 equiv) with out any solvent (Scheme-I). After 3 hours only 27\% of octahydroquinazolinone product was isolated. Increasing the amount of TMSCl (1.5 equiv) did not improve the product yield to a considerable amount. Subsequently, we investigated the effect of different solvents on the reaction rate and as well as yields of the products (Table 1). In protic solvents such as $\mathrm{MeOH}$ or EtOH, the reaction was very slow and resulted in lower product yield. Similar results were obtained in coordinating solvents such as THF, diethyl ether and dimethy ether. On the other hand, conducting the reactions in chlorinated solvents such as dichloromethane and chloroform improved both the reaction rates as well as product yields. After screening for different solvents, acetonitrile / DMF $(6.0 \mathrm{ml}+1.5 \mathrm{ml})$ came out as the solvent of choice, which not only afforded the products in good yield, but also with higher reaction rates $(95 \%$ yield in $1.5 \mathrm{~h}$ ). It is also noticed that the condensation using TMSCl proceeds rapidly and is superior to the reported procedures ${ }^{1,3,4 a-c}$ with respect to reaction time, temperature and yield. This claim is justified through the representative examples, illustrated in Table- 2 , in which the efficiency of TMSCl has been compared with those of recently reported supported Lewis / protic acid catalysts (Table 2).

Table 1. Effect of solvents in the condensation dimedone, benzaldehyde and urea in the presence of TMSCl

\begin{tabular}{cccc}
\hline Entry $^{\mathrm{a}}$ & Solvent & Time $(\mathrm{h})$ & ${\text { Yield }(\%)^{\mathrm{b}}}^{\mathrm{b}}$ \\
\hline 1 & Neat & 3 & 27 \\
2 & Methanol & 5 & 36 \\
3 & Ethanol & 4 & 42 \\
4 & $\mathrm{THF}$ & 3 & 45 \\
5 & $\mathrm{Et}{ }_{2} \mathrm{O}$ & 4 & 49 \\
6 & $\mathrm{DME}_{7}$ & 3 & 52 \\
7 & $\mathrm{CH}_{2} \mathrm{Cl}_{2}$ & 2 & 63 \\
8 & $\mathrm{CHCl}_{3}$ & 2 & 65 \\
9 & $\mathrm{CH}_{3} \mathrm{CN} / \mathrm{DMF}$ & 1.5 & 95 \\
\hline
\end{tabular}

${ }^{a}$ All reactions were performed using benzaldehyde $(10 \mathrm{mmol})$, urea $(15 \mathrm{mmol})$, thiourea (15 $\mathrm{mmol})$, dimedone $(10 \mathrm{mmol})$ and TMSCl $(20 \mathrm{mmol})$ in $\mathrm{CH}_{3} \mathrm{CN} / \mathrm{DMF}(4: 1 \mathrm{ratio})$.

${ }^{\mathrm{b}}$ Isolated yields. 
Table 2. Synthesis of octahydroquinazolinone $\mathbf{4 a}$ using different reagents and reaction conditions

\begin{tabular}{ccccccc}
\hline S.No & Reagent & Solvent & Temp $\left({ }^{\circ} \mathrm{C}\right)$ & Time (h) & Yield (\%) 4a & Ref \\
\hline 1 & Conc. $\mathrm{H}_{2} \mathrm{SO}_{4}$ & Ethanol & 80 & 9.0 & - & $4 \mathrm{a}$ \\
2 & ${\mathrm{Conc} . \mathrm{H}_{2} \mathrm{SO}_{4}}$ & $\mathrm{H}_{2} \mathrm{O}$ & $\mathrm{RT}$ & 3.0 & 85 & 3 \\
3 & $\mathrm{HClO}_{4}-\mathrm{SiO}_{2}(50$ & $\mathrm{CH}_{3} \mathrm{CN}$ & Reflux & 6.0 & 54 & - \\
& $\mathrm{mg}, 0.025 \mathrm{mmol})$ & & & & & \\
4 & Conc. $\mathrm{HCl}$ & Ethanol & Reflux & 6.5 & - & $4 \mathrm{~b}$ \\
5 & $\mathrm{Acid} \mathrm{Alumina}$ & Neat & Reflux & 6.0 & - & 1 \\
6 & $\mathrm{VCl}_{3}$ & $\mathrm{CH}_{3} \mathrm{CN}$ & Reflux & 2.0 & $67-92$ & $4 \mathrm{c}$ \\
7 & $\mathrm{TMSCl}$ & $\mathrm{CH}_{3} \mathrm{CN}$ & Reflux & 5.0 & 15 & - \\
8 & $\mathrm{TMSCl}$ & $\mathrm{CH}_{3} \mathrm{CN} / \mathrm{DMF}$ & Reflux & 1.5 & 95 & - \\
\hline
\end{tabular}

In order to evaluate the generality of the process, several diversified examples illustrating the present method for the synthesis of octahydroquinazolinone $\mathbf{4}$ was studied (Scheme 2, Table 3). The reaction of dimedone $\mathbf{2}$ with various aromatic aldehydes $\mathbf{1}$ bearing electron withdrawing groups (such as nitro, halide) or electron releasing groups (such as N,N-dimethylamino, methyl, hydroxyl; mono, di, or tri methoxy groups), and urea or thiourea 3 was carried out in the presence of TMSCl. The yields obtained were good to excellent without formation of any side products such as 1,8-dioxo-Octahydroxanthenes, which are normally observed under the influence of strong acids ${ }^{4 a}$. The reaction of aromatic aldehydes having electron-withdrawing groups reacted very well at faster rate compared with aromatics aldehydes substituted with electron releasing groups, unlike those of urea, the reactions of thiourea proceeded at lower rate less efficiently to give octahydroquinazolinone (entry 1 and 15, 2 and 16). The results obtained in the current method are illustrated in Table 3. All the products obtained were fully characterized by spectroscopic methods such as IR, ${ }^{1} \mathrm{H}$ NMR, ${ }^{13} \mathrm{C}$ NMR and mass spectroscopy and also by comparison with the reported ${ }^{2 \mathrm{a}, 4 \mathrm{a}}$ spectral data. The simplicity, together with the use of inexpensive, non-toxic and environmentally benign nature of TMSCl under $\mathrm{CH}_{3} \mathrm{CN} / \mathrm{DMF}$ solvent is a remarkable feature of the procedure.

Encouraged by these results we were delighted to observe that the present protocol could safely be extended to the condensation reaction involving equimolar quantity of aldehyde $\mathbf{1}$, dimedone 2. 1-8-dioxo-octahydroxanthenes were obtained in excellent yields (Scheme 3, Table 4) with out the formation of any side products. 
Table 3. TMSCl-Mediated synthesis of various octahydroquinazolinone

\begin{tabular}{|c|c|c|c|c|c|c|c|c|}
\hline \multirow[t]{2}{*}{ Entry } & \multirow[t]{2}{*}{$\mathrm{R}$} & \multirow[t]{2}{*}{$\mathrm{X}$} & \multirow{2}{*}{$\begin{array}{c}\text { Produc } \\
\mathrm{t}^{\mathrm{b}}\end{array}$} & \multirow{2}{*}{$\begin{array}{l}\text { Time } \\
\text { (h) }\end{array}$} & \multirow{2}{*}{$\begin{array}{l}\text { Yield } \\
\% \%^{\mathrm{a}, \mathrm{c}}\end{array}$} & \multicolumn{2}{|c|}{$\operatorname{Mp}\left({ }^{0} \mathrm{C}\right)$} & \multirow[t]{2}{*}{ Ref. } \\
\hline & & & & & & Found & Reported & \\
\hline 1 & $\mathrm{C}_{6} \mathrm{H}_{5}$ & $\mathrm{O}$ & $4 a$ & 1.5 & 95.0 & $292-295$ & 290-291 & $2 a$ \\
\hline 2 & $3-\mathrm{Cl} \mathrm{C} \mathrm{C}_{6} \mathrm{H}_{4}$ & $\mathrm{O}$ & $4 \mathrm{~b}$ & 2.0 & 91.8 & $281-282$ & $282-283$ & $2 a$ \\
\hline 3 & 4- $\mathrm{Cl} \mathrm{C} \mathrm{C}_{6} \mathrm{H}_{4}$ & $\mathrm{O}$ & $4 \mathrm{c}$ & 2.0 & 94.1 & $>300$ & $>300$ & $2 \mathrm{a}, 12$ \\
\hline 4 & $2,4-\mathrm{Cl}_{2} \mathrm{C}_{6} \mathrm{H}_{3}$ & $\mathrm{O}$ & $4 d$ & 2.0 & 82.5 & $268-270$ & - & - \\
\hline 5 & 4- $-\mathrm{BrC}_{6} \mathrm{H}_{4}$ & $\mathrm{O}$ & $4 \mathrm{e}$ & 1.5 & 93.2 & $>300$ & $>300$ & $2 \mathrm{a}$ \\
\hline 6 & 4- $\mathrm{MeC}_{6} \mathrm{H}_{4}$ & $\mathrm{O}$ & $4 \mathrm{f}$ & 2.0 & 87.3 & $>300$ & $>300$ & $4 \mathrm{a}$ \\
\hline 7 & $\mathrm{NMe}_{2} \mathrm{C}_{6} \mathrm{H}_{4}$ & $\mathrm{O}$ & $4 \mathrm{~g}$ & 2.0 & 80.7 & $231-232$ & - & - \\
\hline 8 & $3-\mathrm{OMeC}_{6} \mathrm{H}_{4}$ & $\mathrm{O}$ & $4 \mathrm{~h}$ & 1.5 & 86.2 & $247-248$ & $247-248$ & $4 a$ \\
\hline 9 & $3,4,5-\left(\mathrm{OCH}_{3}\right)_{3} \mathrm{C}_{6} \mathrm{H}_{2}$ & $\mathrm{O}$ & $4 \mathrm{i}$ & 2.0 & 80.5 & $139-140$ & - & - \\
\hline 10 & $\left(\mathrm{CH}_{3}\right)_{2} \mathrm{CH}$ & $\mathrm{O}$ & $4 j$ & 1.5 & 75.8 & $>300$ & - & - \\
\hline 11 & 4-Pyridyl & $\mathrm{O}$ & $4 \mathrm{k}$ & 2.0 & 71.2 & $>300$ & - & - \\
\hline 12 & 4- $\mathrm{NO}_{2} \mathrm{C}_{6} \mathrm{H}_{4}$ & $\mathrm{O}$ & 41 & 1.5 & 84.6 & $304-305$ & - & - \\
\hline 13 & $3-\mathrm{NO}_{2} \mathrm{C}_{6} \mathrm{H}_{4}$ & $\mathrm{O}$ & $4 \mathrm{~m}$ & 1.5 & 78.8 & $299-300$ & $300-301$ & $4 a$ \\
\hline 14 & 4-OHC $6 \mathrm{H}_{4}$ & $\mathrm{O}$ & $4 n$ & 2.5 & 71.7 & $300-302$ & - & - \\
\hline 15 & $\mathrm{C}_{6} \mathrm{H}_{5}$ & $\mathrm{~S}$ & 40 & 2.5 & 76.0 & $284-285$ & - & - \\
\hline 16 & 3- $\mathrm{ClC}_{6} \mathrm{H}_{4}$ & $\mathrm{~S}$ & $4 p$ & 2.5 & 71.6 & $275-276$ & $275-276$ & $2 a$ \\
\hline 17 & 4- $-\mathrm{BrC}_{6} \mathrm{H}_{4}$ & $\mathrm{~S}$ & $4 q$ & 2.5 & 78.4 & $285-286$ & - & - \\
\hline 18 & 4- $\mathrm{OMeC}_{6} \mathrm{H}_{4}$ & $\mathrm{~S}$ & $4 \mathrm{r}$ & 2.5 & 82.0 & $272-275$ & - & - \\
\hline 19 & $3-\mathrm{OMeC}_{6} \mathrm{H}_{4}$ & $\mathrm{~S}$ & $4 \mathrm{~s}$ & 3.0 & 77.5 & $270-272$ & - & - \\
\hline 20 & 4- $\mathrm{MeC}_{6} \mathrm{H}_{4}$ & $\mathrm{~S}$ & $4 \mathrm{t}$ & 2.5 & 81.8 & $273-275$ & - & - \\
\hline 21 & 4- $\mathrm{NMe}_{2} \mathrm{C}_{6} \mathrm{H}_{4}$ & $\mathrm{~S}$ & $4 \mathrm{u}$ & 3.0 & 70.0 & $275-276$ & - & - \\
\hline
\end{tabular}

${ }^{\text {a }}$ All reactions were performed at $10 \mathrm{mmol}$ scale using $20 \mathrm{mmol}$ of $\mathrm{TMSCl}$ in $\mathrm{CH}_{3} \mathrm{CN} / \mathrm{DMF}(6 \mathrm{ml} /$ $1.5 \mathrm{ml}-)$ at reflux temperature $\left(80^{\circ} \mathrm{C}\right)$

${ }^{\mathrm{b}}$ All the products were well characterized by ${ }^{1} \mathrm{H}$ NMR, IR and Mass spectral data.

${ }^{\mathrm{c}}$ Isolated yields.

Trimethylsilyl chloride showed remarkable reactivity as a "hard-soft" reagent and considerably accelerated the reactions. On the basis of all our experimental results, together with literature reports ${ }^{6,10}$, we have proposed the plausible mechanism ${ }^{13}$ for the formation of octahydroquinazolinone 4 in the presence of $\mathrm{TMSCl}$ (Scheme 4). The reaction is believed to precede through the formation of an $\mathrm{N}$-acyliminium ion intermediate 7 from the urea or thiourea and aldehyde 1 precursor in the presence of $\mathrm{TMSCl}$, leading to the formation of octahydroquinazolinone 4 . 
Table 4. TMSCl-Mediated synthesis of various 1,8-dioxo-octahydroxanthenes ${ }^{\text {a }}$

\begin{tabular}{|c|c|c|c|c|c|c|c|c|}
\hline \multirow[t]{2}{*}{ Entry } & \multirow[t]{2}{*}{$\mathrm{Ar}$} & \multirow[t]{2}{*}{ Product ${ }^{b}$} & \multirow[t]{2}{*}{ Time (h) } & \multirow[t]{2}{*}{ Temp $\left({ }^{0} \mathrm{C}\right)$} & \multirow{2}{*}{$\begin{array}{c}\text { Yield }^{\mathrm{c}} \\
(\%)\end{array}$} & \multicolumn{2}{|c|}{$\operatorname{Mp}\left({ }^{0} \mathrm{C}\right)$} & \multirow[t]{2}{*}{ Ref. } \\
\hline & & & & & & Found & Reported & \\
\hline 1 & $\mathrm{C}_{6} \mathrm{H}_{5}$ & $5 a$ & 8 & Reflux & 84.1 & 201-203 & $202-204$ & 11 \\
\hline 2 & 4- $\mathrm{Cl} \mathrm{C}{ }_{6} \mathrm{H}_{4}$ & $5 b$ & 8 & Reflux & 81.0 & $230-232$ & $228-230$ & 11 \\
\hline 3 & $2,4-\mathrm{Cl}_{2} \mathrm{C}_{6} \mathrm{H}_{3}$ & $5 c$ & 8 & Reflux & 74.3 & $254-255$ & $253-254$ & 11 \\
\hline 4 & 4- $\mathrm{Br} \mathrm{C}_{6} \mathrm{H}_{4}$ & $5 \mathrm{~d}$ & 10 & Reflux & 79.2 & $240-242$ & - & - \\
\hline 5 & 3- $\mathrm{OMeC}_{6} \mathrm{H}_{4}$ & $5 e$ & 10 & Reflux & 71.9 & $160-162$ & - & - \\
\hline
\end{tabular}

${ }^{\text {a }}$ All reactions were performed at $10 \mathrm{mmol}$ scale using $20 \mathrm{mmol}$ of $\mathrm{TMSCl}$ in $\mathrm{CH}_{3} \mathrm{CN}(5 \mathrm{ml})$

${ }^{\mathrm{b}}$ All the products were well characterized by ${ }^{1} \mathrm{H}$ NMR, IR and Mass spectral data.

${ }^{\mathrm{c}}$ Isolated yields

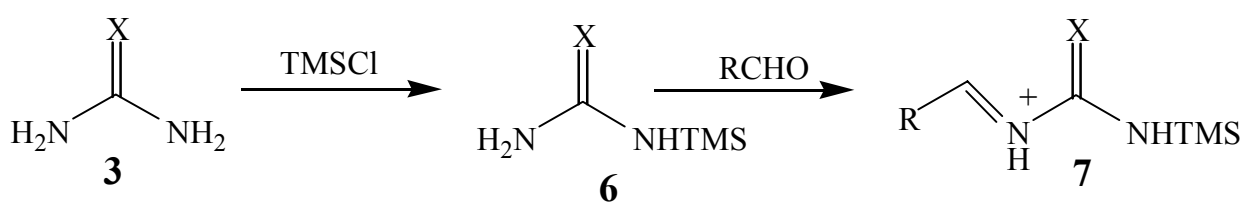<smiles>CC1CC(=O)CC(C)(C)C1</smiles><smiles>[X]C1NC2=C(C(=O)CC(C)(C)C2)C([R])N1</smiles>

4

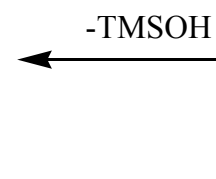

$\mathrm{MSOH}$

9<smiles>[Y]C(N)NC([R])C1=C(O)CC(C)(C)CC1=O</smiles>

8

Scheme 4. Plausible mechanism for the formation of Octahydroquinazolinone 4.

In conclusion, a series of octahydroquinazolinone and 1-8-dioxo-octahydroxanthenes were synthesized efficiently by the condensation of aldehydes $\mathbf{1}$, dimedone $\mathbf{2}$ and urea or thiourea 3 respectively in the presence of $\mathrm{TMSCl}(20 \mathrm{mmol})$ in $\mathrm{CH}_{3} \mathrm{CN} / \mathrm{DMF}$ conditions. The current protocol was also applied successfully for the synthesis of 1,8-dioxo-octahydroxanthenes from aromatic aldehyde and dimedone. The method has ability to tolerate structurally and electronically divergent substituents in aldehydes; variable reaction conditions, shorter reaction times and simple work-up procedure are other advantages. Further, the present procedure is 
readily amenable to large-scale synthesis and the generation of combinatorial octahydroquinazolinones and 1,8-dioxo-octahydroxanthenes.

\section{Experimental Section}

General Procedures. All the commercial reagents and solvents were used without further purification unless otherwise stated. Melting points were recorded on Buchi 535 melting point apparatus and are uncorrected. All the reactions were monitored by thin layer chromatography performed on precoated silica gel $60 \mathrm{~F}_{254}$ plates (Merck). Compounds were visualized with UV light at $254 \mathrm{~nm}$ and $365 \mathrm{~nm}$, iodine and heating plates after dipping in $2 \%$ phosphomolybdic acid in $15 \%$ aq. $\mathrm{H}_{2} \mathrm{SO}_{4}$ solution. NMR spectra were recorded on Varian Unity-400 $\mathrm{MHz}$ and BRUKER AMX $300 \mathrm{MHz}$ spectrometers using tetra methyl silane as an internal standard. ${ }^{13} \mathrm{C}$ NMR was recorded on Varian Unity $100 \mathrm{MHz}$ using $\mathrm{CDCl}_{3}$ as internal standard. Mass spectra were recorded on a VG Micromass $7070 \mathrm{H}$ and Finnigan Mat 1020B mass spectrometers operating at $70 \mathrm{ev}$.

\section{Typical procedure for the synthesis of octahydroquinazolinone}

A solution of benzaldehyde $1 \mathbf{a}(10 \mathrm{mmol})$, dimedone $\mathbf{2 a}(10 \mathrm{mmol})$ and urea or thiourea (15mmol), and MeCN/DMF $(6 \mathrm{ml} / 1.5 \mathrm{ml})$ containing TMSCl $(20 \mathrm{mmol})$ was refluxed $\left(80^{\circ} \mathrm{C}\right)$ till the reaction was completed (monitored by TLC). The solid product $4 \mathbf{a}$ obtained was filtered through a Buchner funnel, washed with $\mathrm{MeCN}(3 \mathrm{x} 5 \mathrm{ml})$ and dried. The compound was recrystallized in ethanol to give pure crystalline product.

The representative spectral data of octahydroquinazolinone derivatives $4 \mathbf{a}-\mathbf{4} \mathbf{u}$ are given below.

7,7-Dimethyl-4-phenyl-4,6,7,8-tetrahydro-1 $\mathrm{H,3H}$-quinazoline-2,5-dione (4a). Mp 292-295 ${ }^{0} \mathrm{C}$, (Lit ${ }^{2 \mathrm{a}} \mathrm{mp}$ 290-291 ${ }^{\circ} \mathrm{C}$ ); IR (KBr) $v_{\max }$ 3317(br), 3258(br), 2961(br), 1709(s), 1673(s), 1608(vs), 1445(w), 1371(s), 1230(s), 761(s), 692(w), 562(w), 486(w), 427(w) $\mathrm{cm}^{-1}$. ${ }^{1} \mathrm{H}$ NMR $\left(300 \mathrm{MHz}, \mathrm{CDCl}_{3}+\mathrm{DMSO}_{6}\right.$ ), $\delta$ 0.97(s, 3H, CMe); 1.10(s, 3H, CMe); 2.18 (q, J=16.1Hz, 2H, $\left.\mathrm{CH}_{2}\right) ; 2.39\left(\mathrm{q}, J=16.8 \mathrm{~Hz}, 2 \mathrm{H}, \mathrm{CH}_{2}\right) ; 5.27(\mathrm{~d}, J=2.8 \mathrm{~Hz}, 1 \mathrm{H}, \mathrm{CH}) ; 7.32-7.21(\mathrm{~m}, 5 \mathrm{H}, \mathrm{Ar}) ; 7.45(\mathrm{~s}$, $1 \mathrm{H}, \mathrm{NH}) ; 9.38(\mathrm{~s}, 1 \mathrm{H}, \mathrm{NH})$; MS (ESI) m/z $271([\mathrm{M}+\mathrm{H}])^{+}$; Anal. Calcd for $\mathrm{C}_{16} \mathrm{H}_{18} \mathrm{~N}_{2} \mathrm{O}_{2}$ : C, 71.09; H, 6.71, N, 10.36. Found: C, 71.16, H, 6.69; N 10.33.

4-(3-Chloro-phenyl)-7,7-dimethyl-4,6,7,8-tetrahydro-1H,3H-quinazoline-2,5-dione (4b). Mp

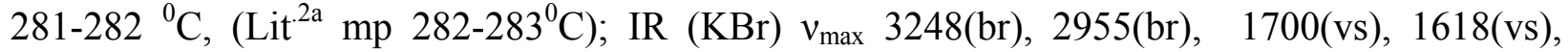
1471(sh), 1376(s), 1236(s), 1048(w), 804(w), 724(br), 563(s), 508(s) cm ${ }^{-1} ; \mathrm{H} \quad \mathrm{NMR}$ $\left(400 \mathrm{MHz}, \mathrm{CDCl}_{3}+\mathrm{DMSO}_{6}\right), \delta$ 0.96(s, 3H, CMe); 1.09(s, 3H, CMe); 2.18 (q, J=16.5Hz, 2H, $\left.\mathrm{CH}_{2}\right) ; 2.39\left(\mathrm{q}, J=18.0 \mathrm{~Hz}, 2 \mathrm{H}, \mathrm{CH}_{2}\right) ; 5.23(\mathrm{~d}, J=2.8 \mathrm{~Hz}, 1 \mathrm{H}, \mathrm{CH}) ; 7.17-7.28(\mathrm{~m}, 4 \mathrm{H}, \mathrm{Ar}) ; 7.66(\mathrm{~s}$, $1 \mathrm{H}, \mathrm{NH}) ; 9.44(\mathrm{~s}, 1 \mathrm{H}, \mathrm{NH})$; MS (ESI) m/z $305([\mathrm{M}+\mathrm{H}])^{+}$. Anal. Calcd for $\mathrm{C}_{16} \mathrm{H}_{17} \mathrm{Cl} \mathrm{N}_{2} \mathrm{O}_{2}$ : C, 63.05; H, 5.62, N, 9.19. Found: C, 63.11, H, 5.62; N 9.23.

4-(4-Chloro-phenyl)-7,7-dimethyl-4,6,7,8-tetrahydro-1H,3H-quinazoline-2,5-dione (4c). $\mathrm{Mp}$ $>300{ }^{0} \mathrm{C}\left(\mathrm{Lit}^{2 \mathrm{a}} \mathrm{mp}>300{ }^{0} \mathrm{C}\right)$; IR (KBr) $v_{\max }$ 3249(br), 2961(br), 1699(vs), 1612(vs), 1488(s), 
1375(s), 1238(s), 809(w), 763(w), 566(s), 509(w) cm ${ }^{-1} ;{ }^{1} \mathrm{H}$ NMR (400MHz, $\left.\mathrm{CDCl}_{3}+\mathrm{DMSO}-\mathrm{d}_{6}\right), \delta$ 0.96(s, 3H, CMe); 1.09(s, 3H, CMe); 2.19(q, J=17.5Hz, 2H, $\left.\mathrm{CH}_{2}\right) ; 2.37\left(\mathrm{q}, J=17.5 \mathrm{~Hz}, 2 \mathrm{H}, \mathrm{CH}_{2}\right.$ ); $5.31(\mathrm{~d}, J=2.9 \mathrm{~Hz}, 1 \mathrm{H}, \mathrm{CH}) ; 7.19-7.30$ (m, 4H, Ar); 7.54(s, 1H, NH); 9.36(s, 1H, NH ); MS (ESI) $\mathrm{m} / \mathrm{z} 305([\mathrm{M}+\mathrm{H}])^{+}$; Anal. Calcd for $\mathrm{C}_{16} \mathrm{H}_{17} \mathrm{Cl} \mathrm{N} \mathrm{O}_{2}$ : C, 63.05; H, 5.62, N, 9.19. Found: C, 63.11, H, 5.61; N 9.20.

4-(2,4-Dichloro-phenyl)-7,7-dimethyl-4,6,7,8-tetrahydro-1H,3H-quinazoline-2,5-dione (4d). Mp 268-270 ${ }^{0} \mathrm{C}$; IR (KBr) $v_{\max }$ 3325(br), 3103(br), 2956(br), 1701(vs), 1627(vs), 1450(m), 1375(s), 1236(s), 862(m), 755(w), 521(w), 464(w), $417 \mathrm{~cm}^{-1} ;{ }^{1} \mathrm{H}$ NMR $\left(400 \mathrm{MHz}, \mathrm{CDCl}_{3}+\right.$ $\left.\mathrm{DMSO}_{6}\right), \delta$ 0.99(s, 3H, CMe); 1.04(s, 3H, CMe); 2.10(q, J=16.1Hz, 2H, $\left.\mathrm{CH}_{2}\right) ; 2.35(\mathrm{q}$, $\left.J=17.6 \mathrm{~Hz}, 2 \mathrm{H}, \mathrm{CH}_{2}\right) ; 5.57(\mathrm{~d}, J=2.9 \mathrm{~Hz}, 1 \mathrm{H}, \mathrm{CH}) ; 7.08-7.19(\mathrm{~m}, 3 \mathrm{H}, \mathrm{Ar}) ; 7.28(\mathrm{~s}, 1 \mathrm{H}, \mathrm{NH})$; 9.45(s, 1H, NH ); ${ }^{13} \mathrm{C}$ NMR (75MHz, DMSO-d $): \delta 192.9(\underline{\mathrm{C}}=\mathrm{O}), 153.3(\mathrm{NC}=\mathrm{O}), 151.0(\mathrm{NC}=\mathrm{C})$, 140.2, 132.9, 132.5, 131.0, 128.9, 127.5 (Ar $\underline{\mathrm{C}}), 105.4$ (OC- $\underline{\mathrm{C}}=\mathrm{C}), 50.6(\underline{\mathrm{C}}-\mathrm{NH}), 49.8\left(\underline{\mathrm{C}} \mathrm{H}_{2}\right), 32.2$ $\left(>\underline{\mathrm{C}}<, \underline{\mathrm{CH}}_{2}\right), 28.6,27.1\left(\underline{\mathrm{CH}}_{3}\right)$; MS (ESI) m/z $340([\mathrm{M}+\mathrm{H}])^{+}$; Anal. Calcd for $\mathrm{C}_{16} \mathrm{H}_{16} \mathrm{Cl}_{2} \mathrm{~N}_{2} \mathrm{O}_{2}$ : C, 56.63; H, 4.75, N, 8.26. Found: C, 56.63, H, 4.74; N 8.26.

4-(4-Bromo-phenyl)-7,7-dimethyl-4,6,7,8-tetrahydro-1 $\mathrm{H}, 3 \mathrm{H}$-quinazoline-2,5-dione (4e). $\mathrm{Mp}$ $>300{ }^{0} \mathrm{C}\left(\mathrm{Lit}^{.2 \mathrm{a}} \mathrm{mp}>300{ }^{0} \mathrm{C}\right)$; IR (KBr) $v_{\max }$ 3249(br), 2961(br), 1708(s), 1612(vs), 1488(sh), 1375(s), 1231(s), $\quad 1011(\mathrm{~s}), \quad 840(\mathrm{w}), \quad 762(\mathrm{br}), \quad 562(\mathrm{w}), \quad 491(\mathrm{w}) \quad \mathrm{cm}^{-1} ; \quad{ }^{1} \mathrm{H} \quad \mathrm{NMR}$ $\left(400 \mathrm{MHz}, \mathrm{CDCl}_{3}+\mathrm{DMSO}_{\mathrm{d}}\right.$ ), $\delta$ 0.95(s, 3H, CMe); 1.08(s, 3H, CMe); 2.16(q, J=16.3Hz, 2H, $\left.\mathrm{CH}_{2}\right) ; 2.37\left(\mathrm{q}, J=17.8 \mathrm{~Hz}, 2 \mathrm{H}, \mathrm{CH}_{2}\right) ; 5.22(\mathrm{~d}, J=2.9 \mathrm{~Hz}, 1 \mathrm{H}, \mathrm{CH}) ; 7.21-7.39(\mathrm{~m}, 4 \mathrm{H}, \mathrm{Ar}) ; 7.56(\mathrm{~s}$, $1 \mathrm{H}, \mathrm{NH}) ; 9.41(\mathrm{~s}, 1 \mathrm{H}, \mathrm{NH})$; MS (ESI) m/z $350([\mathrm{M}+\mathrm{H}])^{+}$; Anal. Calcd for $\mathrm{C}_{16} \mathrm{H}_{17} \mathrm{Br} \mathrm{N}_{2} \mathrm{O}_{2}$ : C, 55.03; H, 4.91, N, 8.02. Found: C, 55.10, H, 4.99; N 8.02.

7,7-Dimethyl-4-p-tolyl-4,6,7,8-tetrahydro-1 $\mathrm{H}$, 3H-quinazoline-2,5-dione (4f). Mp. $>300$ ${ }^{0} \mathrm{C}$,(Lit ${ }^{4 \mathrm{a}} \mathrm{mp}>300{ }^{0} \mathrm{C}$ ); IR (KBr) $v_{\max }$ 3252(br), 2960(br), 1711(s), 1673(vs), 1613(vs), 1420(w), 1374(s), 1233(s), 1045(w), 760(br), 563(w) $\mathrm{cm}^{-1} .{ }^{1} \mathrm{H}$ NMR (500MHz, $\left.\mathrm{CDCl}_{3}+\mathrm{DMSO}_{6}\right), \delta$ 0.97(s, 3H, CMe); 1.09(s, 3H, CMe); 2.16(q, J=16.3Hz, $\left.2 \mathrm{H}, \mathrm{CH}_{2}\right) ; 2.37\left(\mathrm{q}, J=17.9 \mathrm{~Hz}, 2 \mathrm{H}, \mathrm{CH}_{2}\right)$; 2.30(s, $\left.1 \mathrm{H}, \mathrm{CH}_{3}\right), 5.22(\mathrm{~d}, J=1.8 \mathrm{~Hz}, 1 \mathrm{H}, \mathrm{CH}) ; 7.04-7.18(\mathrm{~m}, 4 \mathrm{H}, \mathrm{Ar}) ; 7.29(\mathrm{~s}, 1 \mathrm{H}, \mathrm{NH}) ; 9.28(\mathrm{~s}$, $1 \mathrm{H}, \mathrm{NH}) ; \mathrm{MS}(\mathrm{ESI}) \mathrm{m} / \mathrm{z} 285([\mathrm{M}+\mathrm{H}])^{+}$. Anal. Calcd for $\mathrm{C}_{17} \mathrm{H}_{20} \mathrm{~N}_{2} \mathrm{O}_{2}: \mathrm{C}, 71.81 ; \mathrm{H}, 7.09, \mathrm{~N}$, 9.85; Found: C, 71.82, H, 7.11; N 9.84.

4-(4-Dimethylamino-phenyl)-7,7-dimethyl-4,6,7,8-tetrahydro-1H,3H-quinazoline-2,5-dione (4g). Mp. 231-232 ${ }^{0} \mathrm{C}$; IR (KBr) $v_{\max }$ 3343(br), 3231(br), 2958(br), 1638(m), 1615(vs), 1461(sh), 1375(s), 1242(s), 1191(m), 1146(w), $567 \mathrm{~cm}^{-1} ;{ }^{1 \mathrm{H}} \mathrm{NMR}\left(400 \mathrm{MHz}, \mathrm{CDCl}_{3}+\mathrm{DMSO}_{6}\right), \delta 0.98(\mathrm{~s}$, $3 \mathrm{H}, \mathrm{CMe}) ; 1.09(\mathrm{~s}, 3 \mathrm{H}, \mathrm{CMe}) ; 2.18\left(\mathrm{q}, J=16.1 \mathrm{~Hz}, 2 \mathrm{H}, \mathrm{CH}_{2}\right) ; 2.38\left(\mathrm{q}, J=16.8 \mathrm{~Hz}, 2 \mathrm{H}, \mathrm{CH}_{2}\right) ; 2.54(\mathrm{~s}$, $\left.6 \mathrm{H}, \mathrm{NMe}_{2}\right), 5.25(\mathrm{~d}, J=2.9 \mathrm{~Hz}, 1 \mathrm{H}, \mathrm{CH}) ; 7.32-7.40(\mathrm{~m}, 4 \mathrm{H}, \mathrm{Ar}) ; 7.80(\mathrm{~s}, 1 \mathrm{H}, \mathrm{NH}) ; 9.34(\mathrm{~s}, 1 \mathrm{H}$, $\mathrm{NH}) ;{ }^{13} \mathrm{C}$ NMR $\left(75 \mathrm{MHz}, \mathrm{DMSO}-\mathrm{d}_{6}\right): \delta 193.0(\underline{\mathrm{C}}=\mathrm{O}), 152.8(\mathrm{NC}=\mathrm{O}), 151.6(\mathrm{NC}=\mathrm{C}), 143.6$, 127.6, 119.1 (6xAr므), $106.9(\mathrm{OC} \underline{\mathrm{C}}=\mathrm{C}), 51.4(\underline{\mathrm{C}}-\mathrm{NH}), 49.7\left(\underline{\mathrm{CH}}_{2}\right), 44.4,40.2\left(2 \mathrm{xNCH}_{3}\right)$, 32.2( $\left.>\underline{\mathrm{C}}<, \underline{\mathrm{CH}}_{2}\right), 28.6,27.0\left(\underline{\mathrm{CH}}_{3}\right) ; \mathrm{MS}(\mathrm{ESI}) \mathrm{m} / \mathrm{z} 314([\mathrm{M}+\mathrm{H}])^{+}$Anal. Calcd for $\mathrm{C}_{18} \mathrm{H}_{23} \mathrm{~N}_{3} \mathrm{O}_{2}$ : C, 68.98; H, 7.40, N, 13.41; Found: C, 68.97, H, 7.39; N 13.42.

4-(3-Methoxy-phenyl)-7,7-dimethyl-4,6,7,8-tetrahydro-1 $\mathrm{H}, 3 \mathrm{H}$-quinazoline-2,5-dione (4h). Mp. 247-248 ${ }^{0} \mathrm{C}\left(\mathrm{Lit}^{4 \mathrm{aa}} \mathrm{mp} 247-248{ }^{0} \mathrm{C}\right.$ ); IR (KBr) v $v_{\max }$ 3251(br), 3117(br), 2956(br), 1697(vs), 1618(vs), 1376(s), 1233(sh), 1154(m), 1040(w), 788(w), 732(w), 565, $514 \mathrm{~cm}^{-1}$; ${ }^{1 \mathrm{H}} \mathrm{NMR}$ 
$\left(400 \mathrm{MHz}, \mathrm{CDCl}_{3}+\mathrm{DMSO}_{6} \mathrm{~d}_{6}\right), \delta 0.98(\mathrm{~s}, 3 \mathrm{H}, \mathrm{CMe}) ; 1.09(\mathrm{~s}, 3 \mathrm{H}, \mathrm{CMe}) ; 2.17(\mathrm{q}, J=16.4 \mathrm{~Hz}, 2 \mathrm{H}$, $\left.\mathrm{CH}_{2}\right) ; 2.38\left(\mathrm{q}, J=17.9 \mathrm{~Hz}, 2 \mathrm{H}, \mathrm{CH}_{2}\right) ; 3.75\left(\mathrm{~s}, 3 \mathrm{H}, \mathrm{OCH}_{3}\right), 5.22(\mathrm{~d}, J=2.9 \mathrm{~Hz}, 1 \mathrm{H}, \mathrm{CH}) ; 6.71(\mathrm{dd}$, $J=8.22 \mathrm{~Hz}, 1 \mathrm{H}, \mathrm{Ar}) ; 6.87(\mathrm{~m}, 2 \mathrm{H}, \mathrm{Ar}), 7.18(\mathrm{t}, J=8.22 \mathrm{~Hz}, 1 \mathrm{H}, \mathrm{Ar}) 7.28(\mathrm{~s}, 1 \mathrm{H}, \mathrm{NH}) ; 9.35(\mathrm{~s}, 1 \mathrm{H}$, $\mathrm{NH})$; MS (ESI) m/z $301([\mathrm{M}+\mathrm{H}])^{+}$. Anal. Calcd for $\mathrm{C}_{17} \mathrm{H}_{20} \mathrm{~N}_{2} \mathrm{O}_{3}: \mathrm{C}, 68.98 ; \mathrm{H}, 6.71$, N, 9.33; Found: C, 68.97, H, 6.74; N 9.33.

7,7-Dimethyl-4-(3,4,5-trimethoxy-phenyl)-4,6,7,8-tetrahydro-1H,3H-quinazoline-2,5-dione

(4i). Mp 139-140 ${ }^{0} \mathrm{C}$; IR (KBr) $v_{\max }$ 3371(br), 3236(br), 3114(br), 2943(br), 1639(vs), 1377(s), 1240(s), 1128(s), 100(m)2, 760(w), $577 \mathrm{~cm}^{-1} ;{ }^{1} \mathrm{H}$ NMR (400MHz,CDCl $\left.3+\mathrm{DMSO}_{6} \mathrm{~d}_{6}\right), \delta 1.03(\mathrm{~s}$, $3 \mathrm{H}, \mathrm{CMe}) ; 1.10(\mathrm{~s}, 3 \mathrm{H}, \mathrm{CMe}) ; 2.20\left(\mathrm{q}, J=16.9 \mathrm{~Hz}, 2 \mathrm{H}, \mathrm{CH}_{2}\right) ; 2.39\left(\mathrm{q}, J=16.9 \mathrm{~Hz}, 2 \mathrm{H}, \mathrm{CH}_{2}\right) ; 3.77(\mathrm{~s}$, $\left.3 \mathrm{H}, \mathrm{OCH}_{3}\right), 3.78\left(\mathrm{~s}, 6 \mathrm{H}, \mathrm{OCH}_{3}\right), 5.20(\mathrm{~d}, J=2.9 \mathrm{~Hz}, 1 \mathrm{H}, \mathrm{CH}) ; 6.53(\mathrm{~s}, 2 \mathrm{H}, \mathrm{Ar}) ; 7.28(\mathrm{~s}, 1 \mathrm{H}, \mathrm{NH})$; 9.33(s, 1H, NH); ${ }^{13} \mathrm{C}$ NMR $\left(75 \mathrm{MHz}, \mathrm{DMSO}_{-}\right): \delta 192.9(\underline{\mathrm{C}}=\mathrm{O}), 162.9(\mathrm{NC}=\mathrm{O}), 152.6(\mathrm{NC}=\mathrm{C})$, 139.8, 136.6, 114.1, 106.8, 105.3 (6xAr $\underline{\mathrm{C}}), 103.4(\mathrm{OC}-\underline{\mathrm{C}}=\mathrm{C}), 59.8\left(2 \mathrm{xO}^{\mathrm{C}} \mathrm{H}_{3}\right), 55.6\left(\mathrm{OCH}_{3}\right), 51.7$ $(\underline{\mathrm{C}}-\mathrm{NH}), 49.7\left(\underline{\mathrm{CH}}_{2}\right), 32.1\left(>\underline{\mathrm{C}}<, \underline{\mathrm{CH}}_{2}\right), 28.8,26.5\left(\underline{\mathrm{CH}}_{3}\right) ; \mathrm{MS}(\mathrm{ESI}) \mathrm{m} / \mathrm{z} 361([\mathrm{M}+\mathrm{H}])^{+}$Anal. Calcd for $\mathrm{C}_{19} \mathrm{H}_{24} \mathrm{~N}_{2} \mathrm{O}_{5}$ : C, 63.32; H, 6.71, N, 7.77; Found: C, 63.31, H, 6.71; N 7.76.

4-Isopropyl-7,7-dimethyl-4,6,7,8-tetrahydro- $1 \boldsymbol{H , 3 H}$-quinazoline-2,5-dione $(\mathbf{4 j}) . \mathrm{Mp}>300{ }^{0} \mathrm{C}$; IR (KBr) $v_{\max }$ 3235(br), 3127(br), 2960(br), 1696(vs), 1615(vs), 1380(s), 1239(s), 1151(m), 1062(w), $802 \mathrm{~cm}^{-1} ;{ }^{1} \mathrm{H}$ NMR (400MHz,CDCl $\left.3+\mathrm{DMSO}_{3} \mathrm{~d}_{6}\right), \delta 0.79(\mathrm{~d}, J=7.1 \mathrm{~Hz}, 3 \mathrm{H}), 0.90(\mathrm{~d}$, $J=7.1 \mathrm{~Hz}, 3 \mathrm{H}), \quad 1.06(\mathrm{~s}, 3 \mathrm{H}, \mathrm{CMe}) ; 1.08(\mathrm{~s}, 3 \mathrm{H}, \mathrm{CMe}) ; 2.17\left(\mathrm{q}, J=16.4 \mathrm{~Hz}, 2 \mathrm{H}, \mathrm{CH}_{2}\right) ; 2.32(\mathrm{q}$, $\left.J=17.1 \mathrm{~Hz}, 2 \mathrm{H}, \mathrm{CH}_{2}\right) ; 4.08(\mathrm{~s}, 1 \mathrm{H}, \mathrm{CH}) ; 7.01(\mathrm{~s}, 1 \mathrm{H}, \mathrm{NH}) ; 9.10(\mathrm{~s}, 1 \mathrm{H}, \mathrm{NH}) ;{ }^{13} \mathrm{C} \mathrm{NMR}(75 \mathrm{MHz}$, $\left.\left.\mathrm{CDCl}_{3}+\mathrm{DMSO}_{-}\right)_{6}\right) \delta 191.6(\underline{\mathrm{C}}=\mathrm{O}), 151.5(\mathrm{~N} \underline{\mathrm{C}}=\mathrm{O}), 151.3(\mathrm{NC}=\mathrm{C}), 104.8(\mathrm{OC} \underline{\mathrm{C}}=\mathrm{C}), 52.3(\underline{\mathrm{C}}-$ $\mathrm{NH})$, $48.6\left(\underline{\mathrm{CH}}_{2}\right), 31.9\left(\underline{\mathrm{CH}}_{2}\right) 30.4(>\underline{\mathrm{C}}<), 27.5\left(\underline{\mathrm{CH}}_{3}, \underline{\mathrm{CH}}\right), 25.3,16.9,14.0\left(3 \mathrm{xCH}_{3}\right)$; MS (ESI) $\mathrm{m} / \mathrm{z} 237([\mathrm{M}+\mathrm{H}])^{+}$. Anal. Calcd for $\mathrm{C}_{13} \mathrm{H}_{20} \mathrm{~N}_{2} \mathrm{O}_{2}$ : C, 66.07; H, 8.53, N, 11.85; Found: C, 66.08, $\mathrm{H}, 8.53 ; \mathrm{N} 11.84$.

7,7-Dimethyl-4-pyridin-4-yl-4,6,7,8-tetrahydro-1 $\mathrm{H,3H}$-quinazoline-2,5-dione (4k). $\mathrm{Mp}>300$ ${ }^{0} \mathrm{C}$; IR (KBr) $v_{\max }$ 3122(br), 2962(br), 1689(s), 1637(vs), 1375(s), 1245(s), 1169(m), 786(w), 563 $\mathrm{cm}^{-1} ;{ }^{1} \mathrm{H}$ NMR (400 MHz, CDCl3+DMSO-d $), \delta$ 0.88(s, 3H, CMe); 1.04(s, 3H, CMe); 2.03(q, $\left.J=16.4 \mathrm{~Hz}, 2 \mathrm{H}, \mathrm{CH}_{2}\right) ; 2.38\left(\mathrm{q}, J=17.9 \mathrm{~Hz}, 2 \mathrm{H}, \mathrm{CH}_{2}\right) ; 5.22(\mathrm{~s}, 1 \mathrm{H}, \mathrm{CH}) ; 7.12-7.74(\mathrm{~m}, 4 \mathrm{H}, \mathrm{Ar})$, 8.45(s, 1H, NH); 9.47(s, 1H, NH); ${ }^{13} \mathrm{C}$ NMR (75MHz, DMSO-d $): \delta 192.9(\mathrm{C}=\mathrm{O}), 153.1($ $\mathrm{NC}=\mathrm{O}), 152.5(\mathrm{ArC}), 151.8(\mathrm{NC}=\mathrm{C}), 149.5,121.3(4 \times \mathrm{ArC}), 106.2(\mathrm{OC} \underline{\mathrm{C}}=\mathrm{C}), 59.4(\underline{\mathrm{C}}-\mathrm{NH})$, 50.8, $49.4\left(\underline{\mathrm{CH}}_{2}\right), 32.1(>\underline{\mathrm{C}}<), 28.3,26.5\left(\underline{\mathrm{CH}}_{3}\right)$; MS (ESI) m/z $272([\mathrm{M}+\mathrm{H}])^{+} \cdot$ Anal. Calcd for $\mathrm{C}_{15}$ $\mathrm{H}_{17} \mathrm{~N}_{3} \mathrm{O}_{2} ; \mathrm{C}, 66.40 ; \mathrm{H}, 6.32$, N, 15.41; Found: C, 66.40, H, 6.33; N 15.41.

7,7-Dimethyl-4-(4-nitrophenyl)-4,6,7,8-tetrahydro-1H,3H-quinazoline-2,5-dione (4I). Mp 304-305 ${ }^{0} \mathrm{C}$; IR (KBr) $v_{\max }$ 3323(br), 3244(br), 2963(br), 1671(s), 1623(vs), 1375(m), 1230(s), 829(w), $761 \mathrm{~cm}^{-1} ;{ }^{1} \mathrm{H}$ NMR (400MHz,CDCl $\left.3+\mathrm{DMSO}_{6}\right), \delta$ 0.89(s, 3H, CMe); 1.06(s, 3H, $\mathrm{CMe}) ; 2.16\left(\mathrm{q}, J=16.7 \mathrm{~Hz}, 2 \mathrm{H}, \mathrm{CH}_{2}\right) ; 2.35\left(\mathrm{q}, J=17.4 \mathrm{~Hz}, 2 \mathrm{H}, \mathrm{CH}_{2}\right) ; 5.39(\mathrm{~d}, J=2.8 \mathrm{~Hz}, 1 \mathrm{H}, \mathrm{CH})$; 7.52(d, $J=8.36 \mathrm{~Hz}, 2 \mathrm{H}, \mathrm{Ar}) ; 8.09(\mathrm{~d}, J=8.36 \mathrm{~Hz}, 2 \mathrm{H}, \mathrm{Ar}) ; 7.56(\mathrm{~s}, 1 \mathrm{H}, \mathrm{NH}) ; 9.44(\mathrm{~s}, 1 \mathrm{H}, \mathrm{NH}) ;{ }^{13} \mathrm{C}$ NMR (50MHz, DMSO-d 6$): \delta 193.2(\underline{\mathrm{C}}=\mathrm{O}), 153.3(\mathrm{NC}=\mathrm{O}), 151.8(\mathrm{NC}=\mathrm{C}), 151.7,146.7,127.7$, 123.8 (6x ArC), 106.5(OC드 $\left.=\mathrm{C}), 51.9(\underline{\mathrm{C}}-\mathrm{NH}), 49.8(\underline{\mathrm{CH}})_{2}\right), 32.4\left(>\mathrm{C}<, \underline{\mathrm{CH}}_{2}\right), 28.7,26.9\left(\underline{\mathrm{CH}}_{3}\right)$; MS (ESI) m/z $316([\mathrm{M}+\mathrm{H}])^{+}$. Anal. Calcd for $\mathrm{C}_{16} \mathrm{H}_{17} \mathrm{~N}_{3} \mathrm{O}_{4} ; \mathrm{C}, 60.94 ; \mathrm{H}, 5.43, \mathrm{~N}, 13.33$; Found: C, 60.94, H, 5.44; N 13.32 . 
7,7-Dimethyl-4-(3-nitro-phenyl)-4,6,7,8-tetrahydro-1H,3H-quinazoline-2,5-dione (4m). Mp 299-300 ${ }^{0} \mathrm{C}\left(\mathrm{Lit}^{4 \mathrm{a}} 300-302{ }^{\circ} \mathrm{C}\right) \mathrm{IR}(\mathrm{KBr}) v_{\max } 3117,2956,1697,1618,1377,1233,1153,788 \mathrm{~cm}^{-}$ ${ }^{1} ;{ }^{1} \mathrm{H} \mathrm{NMR}\left(400 \mathrm{MHz}, \mathrm{CDCl}_{3}+\mathrm{DMSO}_{-} \mathrm{d}_{6}\right), \delta 1.06(\mathrm{~s}, 3 \mathrm{H}, \mathrm{CMe}) ; 1.12(\mathrm{~s}, 3 \mathrm{H}, \mathrm{CMe}) ; 2.18(\mathrm{q}$, $\left.J=16.6 \mathrm{~Hz}, 2 \mathrm{H}, \mathrm{CH}_{2}\right) ; 2.43\left(\mathrm{q}, J=16.6 \mathrm{~Hz}, 2 \mathrm{H}, \mathrm{CH}_{2}\right) ; 5.84(\mathrm{~d}, J=2.3 \mathrm{~Hz}, 1 \mathrm{H}, \mathrm{CH}) ; 7.37-7.80(\mathrm{~m}, 4 \mathrm{H}$, Ar); 7.86(s, 1H, NH); 9.58(s, 1H, NH); MS (ESI) m/z $316([\mathrm{M}+\mathrm{H}])^{+}$. Anal. Calcd for $\mathrm{C}_{16} \mathrm{H}_{17} \mathrm{~N}_{3}$ $\mathrm{O}_{4} ; \mathrm{C}, 60.94 ; \mathrm{H}, 5.43, \mathrm{~N}, 13.33$; Found: C, 60.94, H, 5.44; N 13.34 .

4-(4-Hydroxyphenyl)-7,7-dimethyl-4,6,7,8-tetrahydro-1 $\mathrm{H,3} H$-quinazoline-2,5-dione (4n). Mp 300-302 ${ }^{0} \mathrm{C}$; IR (KBr) $v_{\max }$ 3414(br), 3242(br), 2967(br), 1646(vs), 1614(s), 1463(s), 1373(s), 1225(s), 1170(m), 1106(w), 762(w), $504 \mathrm{~cm}^{-1} ;{ }^{1} \mathrm{H}$ NMR (400MHz,CDCl $\left.3+\mathrm{DMSO}_{6}\right), \delta 0.98(\mathrm{~s}$, $3 \mathrm{H}, \mathrm{CMe}) ; 1.09(\mathrm{~s}, 3 \mathrm{H}, \mathrm{CMe}) ; 2.14\left(\mathrm{q}, J=15.8 \mathrm{~Hz}, 2 \mathrm{H}, \mathrm{CH}_{2}\right) ; 2.33\left(\mathrm{q}, J=17.3 \mathrm{~Hz}, 2 \mathrm{H}, \mathrm{CH}_{2}\right) ; 5.14(\mathrm{~d}$, $J=2.2 \mathrm{~Hz}, 1 \mathrm{H}, \mathrm{CH}) ; 6.65(\mathrm{~d}, J=8.65 \mathrm{~Hz}, 2 \mathrm{H}, \mathrm{Ar}), 7.06(\mathrm{~d}, J=8.65 \mathrm{~Hz}, 2 \mathrm{H}, \mathrm{Ar}), 7.45(\mathrm{~s}, 1 \mathrm{H}, \mathrm{NH})$; 9.27(s, 1H, NH); ${ }^{13} \mathrm{C}$ NMR $\left(75 \mathrm{MHz}, \mathrm{DMSO}_{-} \mathrm{d}_{6}\right): \delta 192.9(\underline{\mathrm{C}}=\mathrm{O}), 156.3(\mathrm{NC}=\mathrm{O}), 151.99$ $(\mathrm{NC}=\mathrm{C}), 151.94,135.1,127.3,114.9\left(6 \mathrm{x}\right.$ ArC), $107.8(\mathrm{OCC}=\mathrm{C}), 51.3(\underline{\mathrm{C}}-\mathrm{NH}), 49.8\left(\underline{\mathrm{CH}}_{2}\right), 32.2$ $\left(>\underline{\mathrm{C}}<, \underline{\mathrm{CH}}_{2}\right), 28.7,26.8\left(\underline{\mathrm{CH}}_{3}\right) ; \mathrm{MS}(\mathrm{ESI}) \mathrm{m} / \mathrm{z} 287([\mathrm{M}+\mathrm{H}])^{+}$. Anal. Calcd for $\mathrm{C}_{16} \mathrm{H}_{18} \mathrm{~N}_{2} \mathrm{O}_{3} ; \mathrm{C}$, 67.12; H, 6.34, N, 9.78; Found: C, 67.12, H, 6.33; N 9.77.

7,7-Dimethyl-4-phenyl-2-thioxo-2,3,4,6,7,8-hexahydro-1H- quinazolin-5-azone (4o). Mp. 284-285 ${ }^{0} \mathrm{C}$; IR (KBr) $v_{\max }$ 3259(br), 3175(br), 2957(br), 1618(vs), 1566(s), 1459(s), 1373(s), 1145(m), 1100(sh), 158(w), 690, 553, 516, $427 \mathrm{~cm}^{-1} ;{ }^{1} \mathrm{H}$ NMR (200MHz, $\left.\mathrm{CDCl}_{3}+\mathrm{DMSO}_{6}\right), \delta$ 0.94(s, 3H, CMe); 1.09(s, 3H, CMe); 2.27 (q, J=16.4Hz, 2H, $\left.\mathrm{CH}_{2}\right) ; 2.37\left(\mathrm{~s}, 2 \mathrm{H}, \mathrm{CH}_{2}\right.$ ); 5.25 (d, $J=3.1 \mathrm{~Hz}, 1 \mathrm{H}, \mathrm{CH}) ; 7.18-7.29(\mathrm{~m}, 5 \mathrm{H}, \mathrm{Ar}) ; 9.46(\mathrm{~s}, 1 \mathrm{H}, \mathrm{NH}) ; 10.37(\mathrm{~s}, 1 \mathrm{H}, \mathrm{NH}) ;{ }^{13} \mathrm{C} \mathrm{NMR}$ $\left(75 \mathrm{MHz}, \mathrm{DMSO}-\mathrm{d}_{6}\right): \delta 193.4(\mathrm{C}=\mathrm{O}), 174.5(\mathrm{NC}=\mathrm{O}), 148.5(\mathrm{NC}=\mathrm{C}), 143.2,128.3,127.3,126.2$ (6xArC $), 108.0(\mathrm{OC} \underline{\mathrm{C}}=\mathrm{C}), 52.0(\mathrm{C}-\mathrm{NH}), 49.7\left(\mathrm{CH}_{2}\right), 32.1\left(\mathrm{CH}_{2},>\mathrm{C}<\right), 28.6,26.6\left(\mathrm{CH}_{3}\right)$; MS (ESI) $\mathrm{m} / \mathrm{z} 287([\mathrm{M}+\mathrm{H}])^{+}$. Anal. Calcd for $\mathrm{C}_{16} \mathrm{H}_{18} \mathrm{~N}_{2} \mathrm{O}$ S; C, 67.10; H, 6.33, N, 9.78; Found: C, 67.11, H, 6.33; N 9.77.

4-(3-Chlorophenyl)-7,7-dimethyl-2-thioxo-2,3,4,6,7,8-hexahydro-1H-quinazolin-5-one (4p). Mp 275-276 ${ }^{0} \mathrm{C}$ (Lit $\left.{ }^{2 a} 275-276{ }^{0} \mathrm{C}\right)$ IR (KBr) $v_{\max }$ 3236(br), 2950(br), 1611(vs), 1415(w), 1376(s), 1200(m), 1078(w), 804(w), 713(w), $559 \mathrm{~cm}^{-1} ;{ }^{1} \mathrm{H}$ NMR (200MHz,CDCl 3 + DMSO-d $)$, $\delta$ 0.97(s, 3H, CMe); 1.10(s, 3H, CMe); $2.16\left(\mathrm{q}, J=16.4 \mathrm{~Hz}, 2 \mathrm{H}, \mathrm{CH}_{2}\right) ; 2.38\left(\mathrm{~s}, 2 \mathrm{H}, \mathrm{CH}_{2}\right) ; 5.28$ (d, $J=3.9 \mathrm{~Hz}, 1 \mathrm{H}, \mathrm{CH}) ; 7.27-7.16(\mathrm{~m}, 4 \mathrm{H}, \mathrm{Ar}) ; 9.46(\mathrm{~s}, 1 \mathrm{H}, \mathrm{NH}) ; 10.41(\mathrm{~s}, 1 \mathrm{H}, \mathrm{NH}) ;{ }^{13} \mathrm{C} \mathrm{NMR}$ $\left(75 \mathrm{MHz}, \mathrm{DMSO}-\mathrm{d}_{6}\right): \delta 193.5(\underline{\mathrm{C}}=\mathrm{O}), 174.6(\mathrm{NC}=\mathrm{O}), 148.9(\mathrm{NC}=\mathrm{C}), 145.4,132.8,130.4,127.3$, 126.1, 124.8 (6x Ar$\underline{\mathrm{C}}), 107.4(\mathrm{OC} \underline{\mathrm{C}}=\mathrm{C}), 51.6(\mathrm{C}-\mathrm{NH}), 49.6\left(\underline{\mathrm{CH}_{2}}\right), 32.1\left(>\underline{\mathrm{C}}<, \underline{\mathrm{CH}}_{2}\right), 28.5,26.6$ $\left(\underline{\mathrm{CH}}_{3}\right) ; \mathrm{MS}(\mathrm{ESI}) \mathrm{m} / \mathrm{z} 321.5([\mathrm{M}+\mathrm{H}])^{+}$. Anal. Calcd for $\mathrm{C}_{16} \mathrm{H}_{17} \mathrm{Cl} \mathrm{N} \mathrm{N}_{2} \mathrm{O}$ S; C, 59.90; H, 5.34, N, 8.73; Found: C, 59.90, H, 5.34; N 8.72.

4-(4-Bromophenyl)-7,7-dimethyl-2-thioxo-2,3,4,6,7,8-hexahydro-1H-quinazolin-5-one (4q). Mp 285-286 ${ }^{0} \mathrm{C}$; IR (KBr) $v_{\max }$ 3163(br), 2956(br), 1626(vs), 1570(s), 1459(s), 1375(s), 1201(sh), 1176(m), 1102(m), 1008(m), 781(w), $556 \mathrm{~cm}^{-1} ;{ }^{1} \mathrm{H}$ NMR (200MHz,CDCl 3 + DMSO$\left.\mathrm{d}_{6}\right), \delta 0.88(\mathrm{~s}, 3 \mathrm{H}, \mathrm{CMe}) ; 1.03(\mathrm{~s}, 3 \mathrm{H}, \mathrm{CMe}) ; 2.09\left(\mathrm{q}, J=16.6 \mathrm{~Hz}, 2 \mathrm{H}, \mathrm{CH}_{2}\right) ; 2.29\left(\mathrm{~s}, 2 \mathrm{H}, \mathrm{CH}_{2}\right)$; $5.22(\mathrm{~d}, J=2.9 \mathrm{~Hz}, 1 \mathrm{H}, \mathrm{CH}) ; 7.16(\mathrm{~d}, J=8.7 \mathrm{~Hz}, 2 \mathrm{H}, \mathrm{Ar}) ; 7.35(\mathrm{~s}, J=8.7 \mathrm{~Hz}, 2 \mathrm{H}, \mathrm{Ar}) ; 9.28(\mathrm{~s}, 1 \mathrm{H}$, $\mathrm{NH}) ; 10.30(\mathrm{~s}, 1 \mathrm{H}, \mathrm{NH}) ;{ }^{13} \mathrm{C} \mathrm{NMR}\left(75 \mathrm{MHz}, \mathrm{DMSO}_{-} \mathrm{d}_{6}\right): \delta 193.4(\underline{\mathrm{C}}=\mathrm{O}), 174.5(\mathrm{NC}=\mathrm{O}), 148.7$ $(\mathrm{N} \underline{C}=\mathrm{C}), 142.5,131.7,131.2,128.4,120.4$ (6x Ar $\underline{C}), 107.6(\mathrm{OC} \underline{\mathrm{C}}=\mathrm{C}), 51.6(\underline{\mathrm{C}}-\mathrm{NH}), 49.6\left(\underline{\mathrm{CH}}_{2}\right)$, 
32.1(> $\left.\underline{\mathrm{C}}<, \underline{\mathrm{CH}}_{2}\right), 28.5,26.6\left(\mathrm{CH}_{3}\right)$; MS (ESI) m/z $366([\mathrm{M}+\mathrm{H}])^{+}$. Anal. Calcd for $\mathrm{C}_{16} \mathrm{H}_{17} \mathrm{Br} \mathrm{N}_{2} \mathrm{O}$ S: C, 52.61; H, 4.69, N, 7.67; Found: C, 52.60, H, 4.69; N 7.66.

\section{4-(4-Methoxyphenyl)-7,7-dimethyl-2-thioxo-2,3,4,6,7,8-hexahydro-1 $H$-quinazolin-5-one}

(4r). Mp 272-275 ${ }^{0} \mathrm{C}$; IR (KBr) $v_{\max }$ 3261(br), 3164(br), 2956(br), 1640(vs), 1584(vs), 1375(s), 1252(m), 1168(s), 1023(m), 827(w), 768(w), $551 \mathrm{~cm}^{-1} ;{ }^{1} \mathrm{H} \mathrm{NMR}\left(200 \mathrm{MHz}, \mathrm{CDCl}_{3}+\mathrm{DMSO}_{6}\right)$, $\delta$ 0.96(s, 3H, CMe); 1.10(s, 3H, CMe); 2.14(q, $\left.J=16.0 \mathrm{~Hz}, 2 \mathrm{H}, \mathrm{CH}_{2}\right) ; 3.11\left(\mathrm{~s}, 2 \mathrm{H}, \mathrm{CH}_{2}\right) ; 3.75(\mathrm{~s}$, $\left.3 \mathrm{H}, \mathrm{OCH}_{3}\right), 5.17(\mathrm{~d}, J=2.9 \mathrm{~Hz}, 1 \mathrm{H}, \mathrm{CH}) ; 6.81(\mathrm{~d}, J=8.7 \mathrm{~Hz}, 2 \mathrm{H}, \mathrm{Ar}) ; 7.20(\mathrm{~d}, J=8.7 \mathrm{~Hz}, 2 \mathrm{H}, \mathrm{Ar})$; 9.42(s, 1H, NH); 10.34(s, 1H, NH); ${ }^{13} \mathrm{C}$ NMR (75MHz, DMSO-d 6$): \delta 194.0(\underline{\mathrm{C}}=\mathrm{O}), 174.8$ $(\mathrm{NC}=\mathrm{O}), 159.0(\operatorname{Ar} \underline{\mathrm{C}}), 148.8(\mathrm{NC}=\mathrm{C}), 136.0,128.0,114.2,108.7$ (5x ArC $), 100.3(\mathrm{OCC}=\mathrm{C})$, $\left.55.5\left(\mathrm{OCH}_{3}\right), 52.0(\underline{\mathrm{C}}-\mathrm{NH}), 50.3\left(\underline{\mathrm{CH}}_{2}\right), 32.7(>\underline{\mathrm{C}}<, \underline{\mathrm{CH}})_{2}\right), 29.2,27.2\left(\mathrm{CH}_{3}\right) ; \mathrm{MS}(\mathrm{ESI}) \mathrm{m} / \mathrm{z} 317$ $([\mathrm{M}+\mathrm{H}])^{+} \cdot \mathrm{MS}(\mathrm{ESI}) \mathrm{m} / \mathrm{z} 317([\mathrm{M}+\mathrm{H}])^{+}$. Anal. Calcd for $\mathrm{C}_{17} \mathrm{H}_{20} \mathrm{~N}_{2} \mathrm{O}_{2} \mathrm{~S}: \mathrm{C}, 64.53 ; \mathrm{H}, 6.37, \mathrm{~N}$, 8.85; Found: C, 64.52, H, 6.36; N 8.86.

\section{4-(3-Methoxyphenyl)-7,7-dimethyl-2-thioxo-2,3,4,6,7,8-hexahydro-1H-quinazolin-5-one}

(4s). Mp 270-272 ${ }^{0} \mathrm{C}$; IR (KBr) $v_{\max }$ 3340(br), 3181(br), 2956(br), 1611(vs), 1572(s), 1374(s), 1245, 1147, 1040, 769(w), 704, $567 \mathrm{~cm}^{-1} .{ }^{1} \mathrm{H}$ NMR (400MHz,CDCl 3 + DMSO-d $), \delta 0.97(\mathrm{~s}, 3 \mathrm{H}$, $\mathrm{CMe}) ; 1.11(\mathrm{~s}, 3 \mathrm{H}, \mathrm{CMe}) ; 2.22\left(\mathrm{q},=16.1 \mathrm{~Hz}, 2 \mathrm{H}, \mathrm{CH}_{2}\right) ; 2.39\left(\mathrm{~s}, 2 \mathrm{H}, \mathrm{CH}_{2}\right) ; 3.77\left(\mathrm{~s}, 3 \mathrm{H}, \mathrm{OCH}_{3}\right)$, 5.22(d, $J=3.6 \mathrm{~Hz}, 1 \mathrm{H}, \mathrm{CH}) ; 6.76-7.22(\mathrm{~m}, 4 \mathrm{H}, \mathrm{Ar}) ; 9.51(\mathrm{~s}, 1 \mathrm{H}, \mathrm{NH}) ; 10.41(\mathrm{~s}, 1 \mathrm{H}, \mathrm{NH}) ;{ }^{13} \mathrm{C}$ NMR $\left(75 \mathrm{MHz}, \mathrm{DMSO}-\mathrm{d}_{6}\right): \delta 193.5(\underline{\mathrm{C}}=\mathrm{O}), 174.6(\mathrm{NC}=\mathrm{O}), 159.1(\mathrm{~N}-\underline{\mathrm{C}}=\mathrm{C}), 148.6,144.6,130.0,129.4$, 118.2, $\left.112.3(6 \mathrm{x} \mathrm{ArC}), 107.8(\mathrm{OC} \underline{\mathrm{C}}=\mathrm{C}), 54.8\left(\mathrm{O}_{\underline{C}}\right)_{3}\right), 51.7(\underline{\mathrm{C}}-\mathrm{NH}), 49.7\left(\underline{\mathrm{CH}}_{2}\right), 32.1\left(>\underline{\mathrm{C}}<, \underline{\mathrm{C}} \mathrm{H}_{2}\right)$, 28.6, $26.5\left(2 \mathrm{xCH}_{3}\right)$; MS (ESI) m/z $317([\mathrm{M}+\mathrm{H}])^{+}$. Anal. Calcd for $\mathrm{C}_{17} \mathrm{H}_{20} \mathrm{~N}_{2} \mathrm{O}_{2} \mathrm{~S} ; \mathrm{C}, 64.53 ; \mathrm{H}$, 6.37, N, 8.85; Found: C, 64.54, H, 6.36; N 8.85.

7,7-Dimethyl-2-thioxo-4-p-tolyl-2,3,4,6,7,8-hexahydro-1H-quinazolin-5-one (4t). Mp. 273275; IR (KBr) $v_{\max }$ 3278(br), 2957(br), 1606(vs), 1572(s), 1374(vs), 1261, 1097(m), 816, $759(\mathrm{w}), 580 \mathrm{~cm}^{-1} ;{ }^{1} \mathrm{H}$ NMR (500MHz, $\left.\mathrm{CDCl}_{3}+\mathrm{DMSO}_{6}\right), \delta$ 0.95(s, 3H, CMe); 1.10(s, 3H, $\mathrm{CMe}) ; 2.14\left(\mathrm{q}, J=16.4 \mathrm{~Hz}, 2 \mathrm{H}, \mathrm{CH}_{2}\right) ; 2.36\left(\mathrm{~s}, 2 \mathrm{H}, \mathrm{CH}_{2}\right) ; 2.30\left(\mathrm{~s}, 3 \mathrm{H}, \mathrm{CH}_{3}\right), 5.23(\mathrm{~d}, J=3.1 \mathrm{~Hz}, 1 \mathrm{H}$, $\mathrm{CH})$; 7.18-7.04 (m, 4H, Ar); 9.32(s, 1H, NH); 10.29(s, 1H, NH); ${ }^{13} \mathrm{C}$ NMR (75MHz, DMSO-d 6 ): $\delta 193.4(\mathrm{C}=\mathrm{O}), 174.4(\mathrm{~N}-\mathrm{C}=\mathrm{O}), 148.3(\mathrm{~N}-\mathrm{C}=\mathrm{C}), 140.3,136.5,128.8,126.1(6 \mathrm{x}$ ArC $)$, 108.1(OC드 $=\mathrm{C}), 51.7(\underline{\mathrm{C}}-\mathrm{NH}), 49.7\left(\mathrm{CH}_{2}\right), 32.1\left(>\underline{\mathrm{C}}<, \underline{\mathrm{C}} \mathrm{H}_{2}\right), 28.6,26.6,20.5\left(\underline{\mathrm{CH}}_{3}\right) ; \mathrm{MS}(\mathrm{ESI}) \mathrm{m} / \mathrm{z}$ $301([\mathrm{M}+\mathrm{H}])^{+}$. Anal. Calcd for $\mathrm{C}_{17} \mathrm{H}_{20} \mathrm{~N}_{2} \mathrm{O}$ S: C, 67.97; H, 6.71, N, 9.32; Found: C, 67.96, H, $6.72 ; \mathrm{N} 9.32$.

4-(4-Dimethylamino-phenyl)-7,7-dimethyl-2-thioxo-2,3,4,6,7,8-hexahydro-1 $H$-quinazolin-5one (4u). Mp. 275-276 ${ }^{\circ} \mathrm{C}$; IR (KBr) v $\max 3278($ br), 3180(br), 2957, 1616(vs), 1454(s), 1371, 1235, 807, 764, 556(m), $499 \mathrm{~cm}^{-1} ;{ }^{1} \mathrm{H}$ NMR (200MHz, $\left.\mathrm{CDCl}_{3}+\mathrm{DMSO}_{-} \mathrm{d}_{6}\right), \delta 0.97(\mathrm{~s}, 3 \mathrm{H}, \mathrm{CMe})$; $1.10(\mathrm{~s}, 3 \mathrm{H}, \mathrm{CMe}) ; 2.25\left(\mathrm{q}, J=16.0 \mathrm{~Hz}, 2 \mathrm{H}, \mathrm{CH}_{2}\right) ; 2.37\left(\mathrm{~s}, 2 \mathrm{H}, \mathrm{CH}_{2}\right) ; 2.97\left(\mathrm{~s}, 6 \mathrm{H}, \mathrm{NMe}_{2}\right), 5.18(\mathrm{~d}$, $J=2.9 \mathrm{~Hz}, 1 \mathrm{H}, \mathrm{CH}) ; 6.89-7.20(\mathrm{~m}, 4 \mathrm{H}, \mathrm{Ar}) ; 9.37(\mathrm{~s}, 1 \mathrm{H}, \mathrm{NH}) ; 10.33(\mathrm{~s}, 1 \mathrm{H}, \mathrm{NH}) ;{ }^{13} \mathrm{C} \mathrm{NMR}$ $\left(75 \mathrm{MHz}, \mathrm{DMSO}-\mathrm{d}_{6}\right): \delta 193.5(\underline{\mathrm{C}}=\mathrm{O}), 174.6(\mathrm{~N}-\underline{\mathrm{C}}=\mathrm{O}), 159.1(\mathrm{~N}-\underline{\mathrm{C}}=\mathrm{C}), 148.7,144.6,129.5,118.3$,

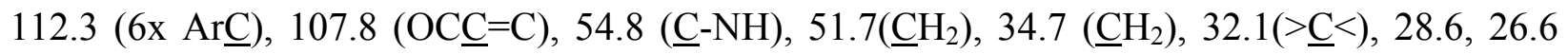
$\left(\underline{\mathrm{CH}}_{3}\right), 18.4\left(2 \mathrm{x} \mathrm{NCH}{ }_{3}\right) ; \mathrm{MS}(\mathrm{ESI}) \mathrm{m} / \mathrm{z} 330([\mathrm{M}+\mathrm{H}])^{+}$. Anal. Calcd for $\mathrm{C}_{18} \mathrm{H}_{23} \mathrm{~N}_{3} \mathrm{O} \mathrm{S} ; \mathrm{C}, 65.62$; H, 7.04, N, 12.75; Found: C, 65.63, H, 7.03; N 12.75. 


\section{Typical procedure for the synthesis of 1,8-dioxooctahydroxanthene}

A solution of benzaldehyde $1 \mathbf{a}(10 \mathrm{mmol})$, dimedone 2a $(20 \mathrm{mmol})$ and $\mathrm{MeCN}(5 \mathrm{ml})$ containing TMSCl $(10 \mathrm{mmol})$ was refluxed till the reaction was completed (monitored by TLC). After completion the reaction mixture was cooled to RT, extracted with EtOAc $(2 \mathrm{x} 25 \mathrm{ml})$. The organic layer was washed with water $(2 \mathrm{x} 20 \mathrm{ml})$, dried over $\mathrm{Na}_{2} \mathrm{SO}_{4}$ and concentrated to obtain crude product. The crude product was crystallized by ethanol to obtain pure 9-aryl-1,8dioxooctahydroxanthene $\mathbf{5 a}$ as white crystalline solid.

The representative spectral ( ${ }^{1} \mathrm{H}$ NMR) data of 1,8-dioxo-octahydroxanthene derivatives 5a-5e are given below.

3,3,6,6-Tetramethyl-9-phenyl-3,4,5,6,7,9-hexahydro- $2 H$-xanthene-1,8-dione (5a). Mp 201$203{ }^{0} \mathrm{C}\left(\mathrm{Lit}^{11} \mathrm{mp} 202-204{ }^{0} \mathrm{C}\right) ;{ }^{1} \mathrm{H}$ NMR $\left(300 \mathrm{MHz}, \mathrm{CDCl}_{3}\right), \delta 0.99\left(\mathrm{~s}, 6 \mathrm{H}, \mathrm{CMe}_{2}\right) ; 1.11(\mathrm{~s}, 6 \mathrm{H}$, $\mathrm{CMe}_{2}$ ); 2.14-2.23(q, $\left.J=15.86 \mathrm{~Hz}, 4 \mathrm{H} 2 \mathrm{xCH}_{2}\right) ; 2.43$ (s, 4H, 2xCH ); 4.68 (s, 1H, CH); 7.04-7.25 (m, 5H, Ar).

9-(4-Chlorophenyl)-3,3,6,6-tetramethyl-3,4,5,6,7,9-hexahydro-2H-xanthene-1,8-dione (5b). Mp 230-232 ${ }^{0} \mathrm{C}$, $\left(\mathrm{Lit}^{11} \mathrm{mp} 228-230{ }^{\circ} \mathrm{C}\right) ;{ }^{1} \mathrm{H}$ NMR (300MHz, $\left.\mathrm{CDCl}_{3}\right), \delta 0.99\left(\mathrm{~s}, 6 \mathrm{H}, \mathrm{CMe}_{2}\right) ; 1.11(\mathrm{~s}$, $\left.6 \mathrm{H}, \mathrm{CMe}_{2}\right) ; 2.10-2.23\left(\mathrm{q}, J=16.61 \mathrm{~Hz}, 4 \mathrm{H} 2 \mathrm{xCH}_{2}\right) ; 2.42$ (s, 4H, 2xCH$) ; 4.63(\mathrm{~s}, 1 \mathrm{H}, \mathrm{CH}) ; 7.14-$ 7.20 (m, 4H, Ar).

9-(2,4-Dichlorophenyl)-3,3,6,6-tetramethyl-3,4,5,6,7,9-hexahydro-2H-xanthene-1,8-dione (5c). Mp 254-255 ${ }^{0} \mathrm{C}\left(\mathrm{Lit}^{11} \mathrm{mp} 253-254{ }^{0} \mathrm{C}\right) ;{ }^{1} \mathrm{H} \mathrm{NMR}\left(300 \mathrm{MHz}, \mathrm{CDCl}_{3}\right), \delta 1.03\left(\mathrm{~s}, 6 \mathrm{H}, \mathrm{CMe}_{2}\right)$; $1.11\left(\mathrm{~s}, 6 \mathrm{H}, \mathrm{CMe}_{2}\right) ; 2.10-2.22\left(\mathrm{q}, J=16.61 \mathrm{~Hz}, 4 \mathrm{H} 2 \mathrm{xCH}_{2}\right) ; 2.40\left(\mathrm{~s}, 4 \mathrm{H}, 2 \mathrm{xCH}_{2}\right) ; 4.85(\mathrm{~s}, 1 \mathrm{H}, \mathrm{CH})$; 7.13-7.43(m, 3H, Ar).

9-(4-Bromophenyl)-3,3,6,6-tetramethyl-3,4,5,6,7,9-hexahydro-2H-xanthene-1,8-dione (5d). Mp 240-242 ${ }^{0} \mathrm{C}$; IR (KBr) $v_{\max }$ 3442(br), 2932(br), 1660(vs), 1585(s), 1362(s), 1274(m), 1201(m), 1138(w), 1047(m), 694(w), 572, $\mathrm{cm}^{-1} ;{ }^{1} \mathrm{H}$ NMR $\left(300 \mathrm{MHz}, \mathrm{CDCl}_{3}\right), 80.99(\mathrm{~s}, 6 \mathrm{H}$, $\left.\mathrm{CMe}_{2}\right) ; 1.11\left(\mathrm{~s}, 6 \mathrm{H}, \mathrm{CMe}_{2}\right) ; 2.10-2.23\left(\mathrm{q}, J=16.61 \mathrm{~Hz}, 4 \mathrm{H} 2 \mathrm{xCH}_{2}\right) ; 2.42\left(\mathrm{~s}, 4 \mathrm{H}, 2 \mathrm{xCH}_{2}\right) ; 4.63$ (s, $1 \mathrm{H}, \mathrm{CH}) ; 7.12(\mathrm{~d}, J=8.30 \mathrm{~Hz}, 2 \mathrm{H}, \mathrm{Ar}) 7.29(\mathrm{~d}, J=8.30 \mathrm{~Hz}, 2 \mathrm{H}, \mathrm{Ar}) ;{ }^{13} \mathrm{C} \mathrm{NMR}(300 \mathrm{MHz}, \mathrm{CDCl} 3): \delta$ $196.3(2 \times \underline{\mathrm{C}}=\mathrm{O}), 162.4(2 \mathrm{x} \mathrm{C}=\underline{\mathrm{C}}-\mathrm{O}), 143.2,131.1,130.1,120.2(6 \mathrm{x} \mathrm{ArC}), 115.2(2 \mathrm{x}(\underline{\mathrm{C}}=\mathrm{C}), 50.6$ (2x $\left.\underline{\mathrm{CH}}_{2}\right), 40.8\left(2 \mathrm{x} \underline{\mathrm{CH}}_{2}\right), 32.1(2 \mathrm{x}>\underline{\mathrm{C}}<), 31.5(\underline{\mathrm{CH}}), 29.2,27.2\left(4 \mathrm{x} \underline{\mathrm{CH}}_{3}\right) ; \mathrm{MS}(\mathrm{ESI}) \mathrm{m} / \mathrm{z} 429$ $([\mathrm{M}+\mathrm{H}])^{+}$Anal. Calcd for $\mathrm{C}_{23} \mathrm{H}_{25} \mathrm{Br} \mathrm{O}_{3}$ : C, 64.34; H, 5.87; Found: C, 64.33, H, 5.87.

9-(3-Methoxyphenyl)-3,3,6,6-tetramethyl-3,4,5,6,7,9-hexahydro-2H-xanthene-1,8-dione (5e). Mp160-162 ${ }^{0} \mathrm{C}$; IR (KBr) $v_{\max }$ 3442(br), 2932(br), 1660(s), 1585(s), 1362(s), 1274, (sh) 1201(m), 1138(m), 1047(w), 694(w), 572, $\mathrm{cm}^{-1} ;{ }^{1} \mathrm{H}$ NMR $\left(300 \mathrm{MHz}, \mathrm{CDCl}_{3}\right), \delta 1.01(\mathrm{~s}, 6 \mathrm{H}$, $\left.\mathrm{CMe}_{2}\right) ; 1.11\left(\mathrm{~s}, 6 \mathrm{H}, \mathrm{CMe}_{2}\right) ; 2.12-2.23\left(\mathrm{q}, J=15.86 \mathrm{~Hz}, 4 \mathrm{H} 2 \mathrm{xCH}_{2}\right) ; 2.43\left(\mathrm{~s}, 4 \mathrm{H}, 2 \mathrm{xCH}_{2}\right) ; 3.37(\mathrm{~s}$, $3 \mathrm{H}, \mathrm{OMe}) ; 4.66(\mathrm{~s}, 1 \mathrm{H}, \mathrm{CH}) ; 6.58-7.10(\mathrm{~m}, 4 \mathrm{H}, \mathrm{Ar}) ;{ }^{13} \mathrm{C} \mathrm{NMR}(300 \mathrm{MHz}, \mathrm{CDCl} 3): \delta 196.2(2 \mathrm{x}$ $\mathrm{C}=\mathrm{O}), 162.2$ (2x C=ㅡ-O), 159.3, 145.6, 128.8, 120.8, 115.5, 114.3(6x ArC), 111.8 2x C=C), $55.0\left(\mathrm{O}_{\underline{C}} H_{3}\right), 50.7\left(2 \mathrm{x} \underline{\mathrm{CH}}_{2}\right), 40.8\left(2 \mathrm{x} \underline{\mathrm{CH}}_{2}\right), 32.1(2 \mathrm{x}>\underline{\mathrm{C}}<), 31.7(\underline{\mathrm{CH}}), 29.1,27.3(4 \mathrm{x} \mathrm{CH})$; MS (ESI) $\mathrm{m} / \mathrm{z} 381([\mathrm{M}+\mathrm{H}])^{+}$. Anal. Calcd for $\mathrm{C}_{24} \mathrm{H}_{28} \mathrm{O}_{4}$ : C, 75.76; H, 7.42; Found: C, 75.76, H, 7.41 . 


\section{Acknowledgements}

We thank Dr. J. S. Yadav, Director, IICT, Hyderabad for constant encouragement and support.

\section{References}

1. Kidwai, M.; Saxena, S.; Khan, M. K. R.; Thukral, S. S. Eur. J. Med. Chem. 2005, 40, 816.

2. (a) Yarim. M.; Sarac, S.; Kilic, S. F.; Erol, K. Il Farmaco. 2003, 58,17. (b) Yarim, M, Sarac, S.; Ertan, M.; Kilic, S. F.; Erol, K. Arzneim-Forsch. 2002, 52, 27.

3. Hassani, Z.; Islami, M. R.; Kalantari, M. Bio. Org., Med. Chem. Lett. 2006, 16, 4479.

4. (a) Tonkikh, N. N.;.Strakovs, A; Petrova, M. V. Chem. Heterocycl.Compds 2004, 40, 43. (b) Candan, M. M.; Kendi, E.; Yarim, M.; Sarac, S.; Ertan, M. Anal. Sci. 2001, 17, 1023. (c) Sabitha, G.; Reddy, G. S. K.; Reddy, K. B.; Yadav, J. S. Tetrahedran Lett. 2003, 44, 6497.

5. Kantevari S.; Srinivasu, V. N. V.; Biradar, D. O.; Nagarapu L. J. Mol. Catalysis A: Chemical 2006, 266, 109.

6. (a) Dilman, A. D.; Loffe, S. L.; Chem. Rev. 2003, 103, 733. (b) Lee, P. H.; Seomoon, D.; Lee, K.; Heo, Y. J. Org. Chem. 2003, 68, 2510. (c) Wang, L. S.; Hollis, T. K. Org.Lett. 2003, 14, 2543. (d) Liu, Y.; Xu, X.; Zang, Y. Tetrahedron 2004, 60, 4867. e) Sabitha, G.; Reddy, G. S. K.; Reddy, K. B.; Yadav, J. S. Synthesis 2004, 263. (f) Sabitha, G.; Reddy, K. S.; Reddy, G. S K.; Fatima, N. Synlett 2005, 2347. (g) Barga, A. L.; Vargas, F.; Sehnem, J. A.; Barga, R. C. J. Org. Chem. 2005, 70, 9021. (h) Xu, L. W.; Xia, C. G. Synthesis 2004, 2191.

7. Wang, T.; Zhang, Z.; Meanwell, N. A. Tetrahedron Lett. 1999, 40, 6745.

8. Zigeuner, G.; Eisenreich, V.; Weichsel, H.; Adam, W. Monatsh. Chem. 1970, 101, 1731.

9. Kappe, C. O. J. Org. Chem. 1997, 62, 7201.

10. Zhu, Y-1.; Huang, S-1.; Pan, Y-j. Eur. J. Org. Chem., 2005, 2354.

11. Jin, T-S.; Zang, J-S.; Xiao, J-C.; Wang, A-Q.; Li, T-S. Synlett 2004, 866.

12. Lin, H.; Zhao, Q.; Xu, B.; Wang, X. J. Mol. Catalysis A: Chemical, 2007 (In Press)

13. We thank the referees and scientific editor for their valuable suggestions. 